\title{
(C) OPEN ACCESS \\ An observational study: associations between nurse-reported hospital characteristics and estimated 30-day survival probabilities
}

\author{
Christine Tvedt, ${ }^{1,2}$ Ingeborg Strømseng Sjetne, ${ }^{1}$ Jon Helgeland, ${ }^{1}$ \\ Geir Bukholm ${ }^{3}$
}

${ }^{1}$ Department of Quality

Measurement and Patient Safety, The Norwegian Knowledge Centre for the Health Services, Oslo, Norway

${ }^{2}$ Institute of Health and Society, University of Oslo, Oslo, Norway ${ }^{3}$ Department of Chemistry, Biotechnology and Food Science, Norwegian University of Life Sciences, Aas, Norway

\section{Correspondence to} Christine Tvedt, The Norwegian Knowledge Centre for the Health Services, PO Box 7004, St. Olavs plass, 0130 Oslo, Norway; christine.raaen.tvedt@Idh.no

Received 20 December 2013 Revised 4 March 2014 Accepted 11 March 2014 Published Online First 12 April 2014

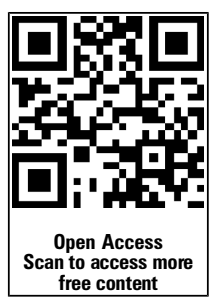

CrossMark

To cite: Tvedt C, Sjetne IS, Helgeland J, et al. BMJ Qual Saf 2014;23:757-764.

\begin{abstract}
Background There is a growing body of evidence for associations between the work environment and patient outcomes. A good work environment may maximise healthcare workers' efforts to avoid failures and to facilitate quality care that is focused on patient safety. Several studies use nurse-reported quality measures, but it is uncertain whether these outcomes are correlated with clinical outcomes. The aim of this study was to determine the correlations between hospital-aggregated, nurseassessed quality and safety, and estimated probabilities for 30-day survival in and out of hospital.
\end{abstract}

Methods In a multicentre study involving almost all Norwegian hospitals with more than 85 beds (sample size=30, information about nurses' perceptions of organisational characteristics were collected. Subscales from this survey were used to describe properties of the organisations: quality system, patient safety management, nurse-physician relationship, staffing adequacy, quality of nursing and patient safety. The average scores for these organisational characteristics were aggregated to hospital level, and merged with estimated probabilities for 30-day survival in and out of hospital (survival probabilities) from a national database. In this observational, ecological study, the relationships between the organisational characteristics (independent variables) and clinical outcomes (survival probabilities) were examined.

Results Survival probabilities were correlated with nurse-assessed quality of nursing.

Furthermore, the subjective perception of staffing adequacy was correlated with overall survival. Conclusions This study showed that perceived staffing adequacy and nurses' assessments of quality of nursing were correlated with survival probabilities. It is suggested that the way nurses characterise the microsystems they belong to, also reflects the general performance of hospitals.

\section{INTRODUCTION}

Healthcare organisations are complex systems that require multifaceted approaches to achieve the goal of patient safety by minimising adverse events that may lead to patient harm. ${ }^{1}$ One approach towards this goal has been to examine how the properties of the organisation can become latent conditions for hazards. Knowledge about how organisational properties work together may be helpful in designing the work environment and the organisation so as to enhance patient safety. The aspect of design may be illustrated by the Swiss cheese model, in which the holes in the Swiss cheese slices represent the aspects of design that allow errors to occur, and how changes in the way the slices are arranged may close these holes and thus prevent those errors from occurring. ${ }^{2}$ Care is provided by frontline healthcare workers, and their ability to ensure quality of care and to discover and close the 'holes' may be facilitated or constrained by the organisational properties. ${ }^{2}$

Donabedian's structure-process-outcome model serves as a framework for identifying organisational properties that have an impact on clinical outcomes. ${ }^{13}$ Organisational structures describe the setting of healthcare delivery. Structure measures, such as nurse staffing levels, teaching status and volume are associated with clinical outcomes, ${ }^{4-8}$ but evidence for impact on clinical outcomes is inconsistent. ${ }^{9}$ Other more changeable and dynamic properties, such as the organisational processes, ${ }^{10}{ }^{11}$ should, therefore, be 
addressed when attempting to design patient-safe organisations. ${ }^{9}$ Processes may be defined and operationalised as the care delivered (clinical process measures) or as organisational processes measures that describe the work environemnt. $^{3} 12{ }^{13}$ It has been suggested that the impact of safety culture vary with the work environment, ${ }^{14}$ and several studies have shown that nursing work environments are associated with clinical and nurse-reported quality. ${ }^{5} 15161718$ Improvements in nurses' perceptions of organisational process measures in their work environment are likely to have an impact on nurse-reported quality. Two studies have shown that nurse-reported quality was associated with clinical outcomes such as hospital standardised mortality rates (HSMR), 30-day mortality, failure to rescue and patient satisfaction. ${ }^{19}{ }^{20}$ However, the evidence for relationships between self-reported quality and clinical outcomes is sparse, and a confirmation of these relationships may enhance the potential to learn from studies that use quality measures reported by healthcare workers as outcome measures. ${ }^{10}$ 17-20

In a previous study, we found that four subscales from a questionnaire measuring nurses' work environment were associated with nurse-assessed patient safety and quality: (1) quality system, (2) staffing adequacy, (3) nurse-physician relationship and (4) patient safety management. ${ }^{11}$ These variables with their interactions were regarded as mutually dependent elements and will be referred to as a 'complex parameter' that represents one way to describe the organisation. In the present study, the complex parameter was applied to explore the associations between nurse-reported work environment, nurse-assessed quality and safety, and clinical outcomes.

\section{Objectives}

The aim of this study was to determine the correlations between hospital-aggregated, nurse-assessed quality and safety measures, and estimated probabilities for 30-day survival in and out of hospital. The complex parameter described in the introduction was applied to examine the associations between nurse-reported organisational process measures and hospital-level clinical outcomes.

\section{METHODS}

\section{Design}

This observational, ecological study combined data from two sources: (1) a multicentre cross-sectional nurse survey in Norwegian hospitals conducted as part of the international project entitled European Nurse Forecasting: Human Resources Planning in Nursing (RN4Cast) and (2) estimated probabilities for 30-day survival in and out of hospital computed by The Norwegian Knowledge Centre for the Health Services based on patient administrative data collected from Norwegian hospitals. Estimated probabilities for 30-day survival in and out of hospital will hereafter be referred to as 'survival probabilities'. Hospital averages for the nurses' responses were computed and merged with hospital-level survival probabilities for 30 hospitals. This study included hospital-level data (survival probabilities) to compare different properties of hospital performance. Since the unit of observation was hospitals, the ecological design was most suitable.

\section{Discharge data}

Survival measures are commonly accepted quality indicators, for example, the HSMR that is corresponding to overall survival described in this study. However, the survival probabilities in the present study include a standardised 30-day follow-up during and after hospital admission. Time is an essential element in mortality measures, and this standardisation prevents that variation in length of stay between the patients compromise the validity of the quality indicator. ${ }^{21}$ In Norway, 30-day survival probabilities are estimated in and out of hospitals for patients admitted for acute myocardial infarction (AMI), stroke and hip fracture. To limit the scope of this study, survival after hip fracture was not included in the present study. The survival probabilities were computed for patients discharged in the period 2005-2009. The discharge data provide information about age, sex, diagnoses, procedure codes, length of stay, departments, municipality of residence, type of admission (acute or elective) and in-hospital mortality. The database was linked to the National Population Register to obtain the exact date of death, so that the cases were followed-up for 30 days from admission during hospitalisation and after discharge (in and out of hospital). Norwegian hospitals' trusts often contain several hospitals, and the survival probabilities were computed at the hospital level. In the present study, survival probabilities after AMI (survival AMI), after stroke (survival stroke) and overall survival were included as clinical outcome measures. Overall survival for 2009 was computed for diagnoses covering $80 \%$ of hospital deaths in hospitals with more than 800 admissions. Inclusion of diagnoses covering $80 \%$ of hospital deaths was made according to procedures for estimation of HSMR. ${ }^{22}$ Cases for AMI and stroke were identified by admission codes according to International Classification of Diseases tenth revision. ${ }^{23}$ Survival probabilities were computed for hospitals with more than 100 cases in the period 2001-2005 and more than 20 for 2009.

A case was defined as starting with first admission of the patient and was followed within the hospital and when transferred to other hospitals (with delay $<8 \mathrm{~h}$ ). To attribute the outcome to the hospitals involved in a case, hospital weighting proportional with the length of stay for each hospital was implemented. ${ }^{21}$ Risk adjustments were made for age, sex, number of admissions (during the previous 2 years) and Charlson comorbidity (during the previous 3 years), and are more thoroughly described elsewhere. ${ }^{21}$ The present study made use of survival probabilities that were published as national quality indicators, and so ethical approval was not necessary. 


\section{Nurse survey}

The Norwegian branch of RN4Cast was conducted during autumn 2009 involving a survey among nurses working bedside in surgical and medical wards in 35 Norwegian hospitals (with more than 85 beds). The data collection procedure is described elsewhere, and the method of data collection and handling was approved by the Data Protection Official for Research. ${ }^{11}$ Two global questions from the RN4Cast questionnaire were used to compare nurses' assessments of quality of nursing and patient safety with clinical outcomes:

- Quality of nursing: 'In general, how would you describe the quality of nursing care delivered to patients on your unit/ward?' (Responses were on a 4-point Likert-type scale, where $1=$ poor, $2=$ fair, $3=$ good and $4=$ excellent)

- Patient safety: 'Please give your department an overall grade on patient safety' (Responses were on a 5-point Likert-type scale, where 1 =failing, 2 =poor, $3=$ acceptable, $4=$ very good and $5=$ excellent)

The complex parameter was based on nurses' descriptions of their work environment and patient safety management. From the instrument Nursing Work Index-Revised (NWI-R), three subscales that are described in table 1 were included (responses were on a 4-point Likert-type scale, where $1=$ strongly disagree, $2=$ somewhat disagree, $3=$ somewhat agree and $4=$ strongly agree). Patient safety management was defined as a composite score including three items that were originally included in the Hospital Survey on Patient Safety Culture (HSOPSC) (responses were on a 5-point Likert-type scale, where $1=$ strongly disagree, $2=$ disagree, $3=$ neither agree nor disagree, $4=$ agree and $5=$ strongly agree) (see table 1$).^{24}$
The Norwegian version of NWI-R was translated and tested according to acknowledged procedures for questionnaire modifications between cultures, and the instrument's reliability and construct validity were assessed in a pilot test. ${ }^{25}$ In a previous paper, we have described the exploratory factor analysis that identified a factor structure of NWI-R for use in a Norwegian context. If more than $50 \%$ of scores in the single items were missing, the scale scores were noted as missing. Internal consistency for the four subscales included in the present study ranged from 0.71 to 0.88 (Cronbach's $\alpha$ ) in the reliability test. Internal consistency of the three items from HSOPSC was 0.72 (Cronbach's $\alpha$ ). ${ }^{11}$

\section{The sample}

Based on recommendations for cut-off points for response rates, four hospitals were excluded because the response rate was below $40 \% .{ }^{26}$ Additionally, one hospital that specialises in cancer care was excluded, and the final sample was 30 hospitals. Responses from 3556 nurses working in surgical or medical wards in these 30 hospitals were included as averaged scores for each hospital. The mean response rate for the present study was $58.9 \%$. Hospitals were categorised into regional university hospitals (university hospitals with national and regional responsibilities), local university hospitals (university hospitals without national responsibilities) and local hospitals (without university functions) that made the reference value in analyses.

\section{Statistical analysis}

The complex parameter and nurse-assessed quality of nursing and patient safety were transformed into a

Table 1 Construction of the subscales: presentation of the single items and the instrument origin (Nursing Work Index-Revised (NWI-R), Hospital Survey on Patient Safety Culture (HSOPSC)

\begin{tabular}{|c|c|}
\hline Subscales & Single items \\
\hline Staffing adequacy (NWI-R) & $\begin{array}{l}\text { Enough time and opportunity to discuss patient care problems with other nurses } \\
\text { Enough registered nurses to provide quality patient care } \\
\text { Enough staff to get the work done }\end{array}$ \\
\hline Nurse-physician relationship (NWI-R) & $\begin{array}{l}\text { Physicians and nurses have good working relationships } \\
\text { A lot of team work between nurses and physicians } \\
\text { Collaboration (joint practice) between nurses and physicians } \\
\text { Physicians value nurses' observations and judgments } \\
\text { Physicians recognise nurses' contributions to patient care } \\
\text { Physicians respect nurses as professionals } \\
\text { Physicians hold nurses in high esteem }\end{array}$ \\
\hline Quality system (NWI-R) & $\begin{array}{l}\text { A clear philosophy of nursing that pervades the patient care environment } \\
\text { Working with nurses who are clinically competent } \\
\text { An active quality assurance programme } \\
\text { A preceptor programme for newly hired registered nurses } \\
\text { Nursing care is based on a nursing model, rather than a medical model } \\
\text { Written, up-to-date nursing care plans for all patients } \\
\text { Patient care assignments that foster continuity of care (ie, the same nurse cares for } \\
\text { the patient from one day to the next) }\end{array}$ \\
\hline Patient safety management (HSOPSC) & $\begin{array}{l}\text { In this unit, we discuss ways to prevent errors from happening again } \\
\text { We are given feedback about changes put into place based on event reports } \\
\text { The actions of hospital management show that patient safety is a top priority }\end{array}$ \\
\hline
\end{tabular}


scale of $0-100$, where high scores represent positive descriptions. Descriptive statistics for the outcome measures in this study are presented as means, 95\% CIs, minimum and maximum. Means and 95 CIs for the nurse-reported measures are described per hospital type. The nurse-assessed quality and safety measures and survival probabilities were included in a correlation analysis, from which the results are reported as Pearson's correlation coefficients and $\mathrm{p}$ values. The complex parameter and hospital type were included in a regression analysis to examine the associations with nurse-assessed quality and safety, and survival probabilities that are reported as regression coefficients and $\mathrm{p}$ values. Initial univariate analyses were not performed to determine which variables to include in the logistic model, since the variables were included as subcomponents of a complex parameter based on findings from a previous paper. ${ }^{11}$ In the additional regression analysis, hospitallevel nurse-to-patient ratios collected from Statistics Norway were included (number of nurse-years per 10000 patient days). ${ }^{27}$

SPSS V.18.0 Software (SPSS, Chicago, Illinois, USA) for Windows was used for correlation analysis, and $\mathrm{R}$ 2.15.1, free software available at (http://www. r-project.org/), was used to conduct the multivariate stepwise regression (conditional backwards with the Akaike information criterion) on the hospital-level dataset.

\section{RESULTS}

Survival probabilities varied between hospitals, and the hospital mean for overall survival was 95.1\% (95\% CI $94.8 \%$ to $95.4 \%$, minimum $=93.8 \%$, maximum $=96.2 \%$ ). The hospital mean for survival AMI was $87.1 \%$ (95\% CI $86.2 \%$ to $88.1 \%$, minimum $=83.6 \%$, maximum $=93.8 \%$ ) and for survival stroke $85.5 \%$ (95\% CI $84.4 \%$ to $86.7 \%$, minimum $=74.8 \%$, maximum $=89.1 \%$ ). The mean score for nurses' assessments of quality of nursing was 66.9 $(95 \%$ CI 65.2 to $68.7, \quad$ minimum $=54.8$, maximum $=76.0$ ). The mean score for patient safety was $60.3(95 \%$ CI 58.9 to $61.8, \quad$ minimum $=48.5$, maximum $=67.4$ ).

Most of the hospitals were categorised as local $(n=22)$, but three hospitals were considered to be local university hospitals and further five were regional university hospitals. The variation in independent variables is presented per hospital type in table 2.

The bivariate correlation analysis showed that nurse-assessed quality of nursing was correlated with nurse-assessed patient safety, overall survival and survival after AMI (table 3).

Nurses' assessments of patient safety were not correlated with the survival probabilities, and survival after stroke was not correlated with any of the other measures (table 3).
The multivariate stepwise regression analysis showed that nurses' assessments of staffing adequacy were positively associated with overall survival, while there was a negative association between patient safety management and overall survival (table 4). A positive perception of staffing adequacy and regional university hospitals was associated with higher survival after AMI. There was no association between the organisational measures and survival after stroke. Staffing adequacy was associated with the two nurse-assessed outcomes, and patient safety management was associated with patient safety (table 4). The variables that remained significant at 5\% level in the linear stepwise regression are presented in the table.

Nurse-reported staffing adequacy was associated with three of the outcome measures, and so we repeated the model analysis and included nurse-to-patient ratios based on figures from official statistics (ie, number of nurse man-years per 10000 patient days, in 2009). ${ }^{27}$ This did not result in significant changes.

\section{DISCUSSION}

In the present study, we found that overall survival is correlated with nurse-assessed quality of nursing. Furthermore, one of the diagnosis-specific survival measures, survival after AMI, is correlated with overall survival and quality of nursing. The subjective impression of staffing adequacy was correlated with overall survival, whereas the nurse-to-patient ratio was not associated with survival probabilities.

Hospitals are hierarchical systems consisting of wards that represent microsystems in which interactions (or care delivery) take place between healthcare workers and patients. According to Nelson et al, ${ }^{28}$ these microsystems are 'essential building blocks that combine to form the care continuum'. In the present study, quality of care at the microsystem level was reflected in nurses' assessments of quality and, to some extent, nurses' assessments of patient safety, whereas quality of care at the hospital level was reflected in survival probabilities. Nurses' assessments of quality of nursing in their wards are strongly correlated with overall survival, while their assessments of patient safety are not. The presence of a relationship between quality of nursing and overall survival is supported by a similar study, from whose results it was concluded that staff feedback is weakly associated with HSMR. ${ }^{20}$ The question regarding patient safety called for the nurses' perceptions at the department level, which is one level above their ward level (a department in Norwegian hospitals most often consists of several wards). The department is a more distant entity, and the question about patient safety may reflect the nurses' judgement of the patient safety systems administered by people outside their own ward and their own microsystem. ${ }^{29}{ }^{30}$ This may also be one way to understand why patient safety management was negatively associated with overall survival. 
Table 2 Mean and Cls for organisational characteristics per hospital type

\begin{tabular}{|c|c|c|c|c|c|c|}
\hline Hospital type & & $\begin{array}{l}\text { Staffing } \\
\text { adequacy* }\end{array}$ & $\begin{array}{l}\text { Nurse-physician } \\
\text { relationship* }\end{array}$ & $\begin{array}{l}\text { Quality } \\
\text { system* }\end{array}$ & $\begin{array}{l}\text { Patient safety } \\
\text { management* }\end{array}$ & $\begin{array}{l}\text { Nurse-to-patient } \\
\text { ratiot }\end{array}$ \\
\hline \multirow[t]{3}{*}{ Local hospital $(n=22)$} & Mean & 43.9 & 68.0 & 52.0 & 55.1 & 51.3 \\
\hline & $\begin{array}{l}\text { Lower } \\
95 \% \mathrm{Cl}\end{array}$ & 40.5 & 66.2 & 49.3 & 52.9 & 47.2 \\
\hline & $\begin{array}{l}\text { Upper } \\
95 \% \mathrm{Cl}\end{array}$ & 47.3 & 69.7 & 54.7 & 57.3 & 55.1 \\
\hline \multirow{3}{*}{$\begin{array}{l}\text { Local university } \\
\text { hospital }(n=3)\end{array}$} & Mean & 42.0 & 65.1 & 53.1 & 53.7 & 53.2 \\
\hline & $\begin{array}{l}\text { Lower } \\
95 \% \mathrm{Cl}\end{array}$ & 28.1 & 57.9 & 48.3 & 47.1 & 41.6 \\
\hline & $\begin{array}{l}\text { Upper } \\
95 \% \mathrm{Cl}\end{array}$ & 55.9 & 72.3 & 57.8 & 60.2 & 64.8 \\
\hline \multirow{3}{*}{$\begin{array}{l}\text { Regional university } \\
\text { hospital }(n=5)\end{array}$} & Mean & 49.0 & 65.8 & 51.6 & 54.9 & 68.2 \\
\hline & $\begin{array}{l}\text { Lower } \\
95 \% \mathrm{Cl}\end{array}$ & 37.0 & 62.9 & 43.5 & 48.1 & 52.7 \\
\hline & $\begin{array}{l}\text { Upper } \\
95 \% \mathrm{Cl}\end{array}$ & 61.2 & 68.7 & 59.6 & 61.6 & 83.7 \\
\hline
\end{tabular}

*The original 4- or 5-point Likert scales have been transformed into 0-100 scale, where high scores represent positive descriptions in this table. tNumber of nurse-years per 10000 patient days.

Another way to understand this odd result is that patient safety may have had a high priority in the wards where staffing was perceived as adequate. In wards where the attention towards patient safety is high, the attitudes towards safety and quality among the respondents might have been more conscious and, therefore, more critical. Moreover, the cross-sectional design of this study did not allow adjustments for variations over time. If interventions prior to the nurse survey changed the respondents' perceptions of the work environment, this may not have had impact on the survival probabilities that are based on data from a long period of time.

We also examined the survival of two diagnosis groups: AMI and stroke. Survival after AMI was correlated with overall survival and with nurses' assessments of quality of care. The different processes implemented for the treatment of AMI and stroke may explain this finding. Survival after stroke is dependent on acute functions in primary healthcare, a multifaceted intervention within specialist healthcare and a good rehabilitation plan in primary healthcare after discharge from hospital. A crucial factor is the time from symptom onset to medical treatment and admission to hospital. The accessibility of healthcare, distance from the hospital, and patient paths within and between the hospitals are perhaps more important organisational aspects for survival after stroke. The management of AMI patients was revised in Norway from 2000, and standardised procedures are now followed for the acute care provided by hospitals managing these patients. This is reflected in better survival data for AMI in a comparative national survey, as recorded in the Organization for Economic Co-operation and Development's document 'Health at a Glance'. ${ }^{31}$ It may not be surprising that nurses assess the quality of care as being high in those hospitals with well-functioning acute care. However, a limitation of the present study is that the nurses' assessments of quality of care was not broken down and stratified according to AMI and stroke wards. Therefore, it was not possible to examine the correlation between quality of care and survival at the ward level. Another limitation of the present study is that responses from nurses working in critical care units in which these patients may be cared for at their most vulnerable state, was not included.

Table 3 Correlation coefficients between nurse-assessed outcomes and estimated survival probabilities

\begin{tabular}{lllll}
\hline & $\begin{array}{l}\text { Survival after AMI } \\
\text { Pearson's correlation }(\mathrm{p} \text { value) }\end{array}$ & $\begin{array}{l}\text { Survival after stroke } \\
\text { Overall survival }\end{array}$ & Quality of nursing \\
\hline Survival after AMI & - & & \\
Survival after stroke & $0.01(0.960)$ & - & - & \\
Overall survival & $0.56(0.001)$ & $0.30(0.106)$ & $0.49(0.006)$ & - \\
Quality of nursing & $0.40(0.028)$ & $0.00(0.990)$ & $0.32(0.088)$ & $0.82(<0.001)$ \\
Patient safety & $0.30(0.114)$ & $0.02(0.918)$ & & - \\
\hline
\end{tabular}

$\mathrm{AMI}$, acute myocardial infarction. 
Table 4 Multivariate stepwise regression model (backwards conditional) showing associations between organisational characteristics and nurse-assessed outcomes and estimated survival probabilities

\begin{tabular}{llllll}
\hline & \multicolumn{2}{l}{$\begin{array}{l}\text { Overall survival } \\
\text { Regression coefficient ( } \mathrm{p} \text { value })\end{array}$} & Survival after stroke & Quality of nursing & Patient safety \\
\hline Patient safety management & $-0.09(0.048)^{*}$ & - & - & - & $0.36(0.011)^{\star}$ \\
Staffing adequacy & $0.09(0.002)^{\star *}$ & $0.08(0.079)$ & - & $0.44(<0.001)^{\star *}$ & $0.24(0.005)^{\star *}$ \\
Nurse-physician relationship & - & - & - & - & - \\
Quality system & - & - & - & - & - \\
Local university hospital $\dagger$ & - & $1.10(0.346)$ & - & - & - \\
Regional university hospital $\dagger$ & - & $4.05(<0.001)^{\star *}$ & - & - & - \\
Adjusted $R^{2}$ & 0.30 & 0.45 & - & 0.57 & 0.79 \\
\hline
\end{tabular}

* Significant at the $0.01 \%$ level.

** Significant at the $0.05 \%$ level.

tReference value is all other hospitals.

AMI, acute myocardial infarction.

The finding that survival after AMI was higher in regional university hospitals than in the other hospital types may indicate that the former are pioneers at improving the processes of care, which consequently reduces mortality. ${ }^{32}$ The introduction of percutaneous coronary intervention in regional university hospitals during the period studied may have had a major effect on survival for patients with AMI. Higher volume, higher personnel ratios and other structural features of teaching hospitals may also explain the better outcomes. However, we did not find the association between the nurse-to-patient ratio and survival that has been found in other studies. $^{5}$ 33-35 Hence, the association between nurse-reported staffing adequacy and overall survival indicates that the way resources are organised impacts patient outcomes. It is therefore important to emphasise that nurse-reported staffing adequacy is a composite variable that reflects factors additional to simply the number of nurses. This finding is consistent with another study that showed that nurse-reported staffing adequacy is not correlated with the nurse-to-patient ratio. ${ }^{36}$ On the other hand, it has been shown that nurse-reported staffing adequacy is associated with the accessibility of assistive personnel, ${ }^{37}$ and qualitative interviews indicate that the content of staffing adequacy includes how nurses assess the personnel mix, the cohesiveness of the staff, the delivery systems and 'knowing' the patients. ${ }^{38}$ In a recent literature review, perceived staffing adequacy was associated with clinical outcomes such as mortality, failure to rescue and adverse events. ${ }^{17}$ From our perspective, together these results emphasise the importance of having organisational processes that facilitate the quality of care and patient safety. This is particularly important considering our findings indicating that the way personnel resources are organised and allocated is more vital for quality and safety than the number of personnel. However, further research is required on what makes healthcare personnel perceive staffing as adequate.
Several studies have found associations between clinical outcomes and other organisational process measures, such as the nurse-physician relationship, the general work environment and educational level. 18 34 $39-40$ The finding that staffing adequacy was the only process measure of significance in the present study may be attributable to several factors. First, we used a selection of organisational process measures identified in a previous study; the measures used in other studies may not be compatible with our complex parameter. Second, other studies used patients as units of analysis; our ecological approach provides another perspective and involves a relatively small number of cases. Third, the outcome measures in the present study, survival probabilities, were weighted for hospital length of stay, and followed the cases after hospital discharge. This approach differs from similar studies using in-hospital mortality, or mortality during 30 days in hospital.

\section{Strengths and limitations}

The use of mortality measures for comparison purposes has been criticised because the different ways to calculate mortality may provide 'large variation in hospital ranking and outlier detection'. ${ }^{21}$ Transfer of patients between hospitals might explain this problem, and the survival probabilities used in the present study have been recommended as indicators for hospital performance. ${ }^{21}$ Another strength of our study is the comprehensiveness of the nurse survey, for which there was a response rate of $58.9 \%$, and which involved the majority of Norwegian general hospitals. A comparative study has shown that nurses' ratings of their work environment, staffing levels, educational level and skill mix are generally high in Norway compared with other countries. ${ }^{41}$ This makes Norway a unique arena in which to explore the issues of patient safety, because the findings may point to organisational properties that are crucial for patient safety, and that may be concealed in other contexts.

Ecological studies are criticised because data at the individual level are aggregated to the group level. 
However, as the intention of this study was to compare hospital performance including hospital-level data, the ecological design was necessary.

The survey method is a cost-effective way of investigating the organisational properties of healthcare organisations, and the subjective description of quality made by nurses appears to be a valid measure. ${ }^{19} 20$ However, the healthcare workers' reports on quality and safety may be biased for several reasons, and the discrepancy between healthcare workers' assessments and clinical outcomes may be great. ${ }^{17}$ Still, nurse-assessed quality has been used to describe the role of nursing in hospitals, and to identify properties of high-performing work systems. Our study may serve as a support for studies using healthcare workers assessments as outcome measures to examine organisational properties that promote quality of care and patient safety.

\section{CONCLUSION}

In this study, perceived staffing adequacy, but not nurse-patient ratio was associated with overall survival. This result suggests that the way resources are organised is a critical factor to achieve the goal of quality and safety. Furthermore, nurses' assessments of quality of nursing were correlated with overall survival and survival after AMI, suggesting that the way nurses characterise the microsystems they belong to also reflects the general performance of hospitals.

Acknowledgements The authors would like to thank all the Norwegian nurses who completed the nurse survey.

Contributors All the authors contributed to design of the study, and have read and approved the final version of the manuscript. CT drafted the manuscript and conducted the statistical analyses. CT and ISS carried out data collection using the nurse survey, and ISS contributed to manuscript revision. JH contributed with statistical advice and expertise concerning the survival probabilities. GB had the main responsibility for the conception and design of the study and revisions of the manuscript drafts.

Funding Data were collected by Norwegian Nurses' Organisation and the Norwegian Knowledge Centre for the Health Services in collaboration. CT was supported by a grant from Norwegian Nurses' Organisation.

\section{Competing interests None.}

Ethical approval Data Protection Official for Research approved the method of data collection and handling of the nurse survey.

Provenance and peer review Not commissioned; externally peer reviewed.

Open Access This is an Open Access article distributed in accordance with the Creative Commons Attribution Non Commercial (CC BY-NC 3.0) license, which permits others to distribute, remix, adapt, build upon this work noncommercially, and license their derivative works on different terms, provided the original work is properly cited and the use is non-commercial. See: http://creativecommons.org/licenses/by$\mathrm{nc} / 3.0 /$

\section{REFERENCES}

1 Battles JB, Lilford RJ. Organizing patient safety research to identify risks and hazards. Qual Saf Health Care 2003;12 (Suppl 2):ii2-7.
2 Reason J. Human error: models and management. BMJ 2000;320:768-70.

3 Donabedian A. Selecting approaches to assessing performance. In: Bashshur R.ed An introduction to quality assurance in health care. New York: Oxford University Press, 2003, 45-56.

4 Vartak S, Ward MM, Vaughn TE. Do postoperative complications vary by hospital teaching status? Med Care 2008;46:25-32.

5 Kane RL, Shamliyan TA, Mueller C, et al. The Association of Registered Nurse Staffing Levels and Patient Outcomes: Systematic Review and Meta-Analysis. Med Care 2007;45:1195-204.

6 Thornlow DK, Stukenborg GJ. The association between hospital characteristics and rates of preventable complications and adverse events. Med Care 2006;44:265-9.

7 Ayanian JZ, Weissman JS. Teaching hospitals and quality of care: a review of the literature. Milbank $Q 2002$; 80:569-93, v.

8 Birkmeyer JD, Siewers AE, Finlayson EV, et al. Hospital volume and surgical mortality in the United States. N Engl J Med 2002;346:1128-37.

9 Shekelle PG, Pronovost PJ, Wachter RM, et al. Advancing the science of patient safety. Ann Intern Med 2011; 154:693-6.

10 Djukic M, Kovner CT, Brewer CS, et al. Work environment factors other than staffing associated with nurses' ratings of patient care quality. J Nurs Adm 2012;42(10 Suppl):S17-26.

11 Tvedt C, Sjetne IS, Helgeland J, et al. A cross-sectional study to identify organisational processes associated with nurse-reported quality and patient safety. BMJ Open 2012;2; e001967.

12 Donabedian A. An introduction to quality assurance in health care. New York: Oxford University Press, 2003.

13 Carayon P, Schoofs HA, Karsh BT, et al. Work system design for patient safety: the SEIPS model. Qual Saf Health Care 2006;15(Suppl 1):i50-8.

14 Clarke SP, Aiken LH. More nursing, fewer deaths. Qual Saf Health Care 2006;15:2-3.

15 Gunnarsdottir S, Clarke SP, Rafferty AM, et al. Front-line management, staffing and nurse-doctor relationships as predictors of nurse and patient outcomes. a survey of Icelandic hospital nurses. Int J Nurs Stud 2009;46:920-7.

16 Purdy N, Spence Laschinger HK, Finegan J, et al. Effects of work environments on nurse and patient outcomes. J Nurs Manag 2010;18:901-13.

17 Bae SH. Assessing the relationships between nurse working conditions and patient outcomes: systematic literature review. J Nurs Manag 2011;19:700-13.

18 Aiken LH, Cimiotti JP, Sloane DM, et al. Effects of nurse staffing and nurse education on patient deaths in hospitals with different nurse work environments. Med Care 2011;49:1047-53.

19 McHugh MD, Stimpfel AW. Nurse reported quality of care: a measure of hospital quality. Res Nurs Health 2012;35:566-75.

20 Pinder RJ, Greaves FE, Aylin PP, et al. Staff perceptions of quality of care: an observational study of the NHS Staff Survey in hospitals in England. BMJ Qual Saf 2013;22:563-70.

21 Kristoffersen DT, Helgeland J, Clench-Aas J, et al. Comparing hospital mortality-how to count does matter for patients hospitalized for acute myocardial infarction (AMI), stroke and hip fracture. BMC Health Serv Res 2012;12:364.

22 Heijink R, Koolman X, Pieter D, et al. Measuring and explaining mortality in Dutch hospitals; the hospital 
standardized mortality rate between 2003 and 2005. BMC Health Serv Res 2008;8:73.

23 World Health Organization. [International classification of diseases]. 2010

24 Blegen MA, Gearhart S, O'Brien R, et al. AHRQ's hospital survey on patient safety culture: psychometric analyses. J Patient Saf 2009;5:139-44.

25 Sjetne IS, Tvedt C, Squires A. Måleinstrumentet "The Nursing Work Index-Revised” -oversettelse og utprøving av en norsk versjon. ["The Nursing Work Index-Revised" -Instrument translation and assessment of a Norwegian version]. Sykepleien Forskning 2011;6:358-65.

26 Kramer M, Schmalenberg C, Brewer BB, et al. Accurate assessment of clinical nurses' work environments: response rate needed. Res Nurs Health 2009;32:229-40.

27 Statistics Norway. Statbank. http://statbank ssb no/statistikkbanken/ [ 2009 URL:http://statbank.ssb.no/statistikkbanken/

28 Nelson EC, Batalden PB, Huber TP, et al. Microsystems in health care: Part 1. Learning from high-performing front-line clinical units. Jt Comm J Qual Improv 2002;28:472-93.

29 Krogstad U, Hofoss D, Veenstra M, et al. Hospital quality improvement in context: a multilevel analysis of staff job evaluations. Qual Saf Health Care 2005;14:438-42.

30 Mohr JJ, Barach P, Cravero JP, et al. Microsystems in health care: Part 6. Designing patient safety into the microsystem. Jt Comm J Qual Saf 2003;29:401-8.

31 Organisation for Economic Co-operation and Development. Health at a Glance 2009. 2009.

32 Rivard PE, Elixhauser A, Christiansen CL, et al. Testing the association between patient safety indicators and hospital structural characteristics in VA and nonfederal hospitals. Med Care Res Rev 2010;67:321-41.

33 Tourangeau AE, Giovannetti P, Tu JV, et al. Nursing-related determinants of 30-day mortality for hospitalized patients. Can J Nurs Res 2002;33:71-88.

34 Estabrooks CA, Midodzi WK, Cummings GG, et al. The impact of hospital nursing characteristics on 30-day mortality. Nurs Res 2005;54:74-84.

35 Tourangeau AE, Doran DM, McGillis HL, et al. Impact of hospital nursing care on 30-day mortality for acute medical patients. J Adv Nurs 2007;57:32-44.

36 Kalisch BJ, Friese CR, Choi SH, et al. Hospital nurse staffing: choice of measure matters. Med Care 2011; 49:775-9.

37 Mark BA, Salyer J, Harless DW. What explains nurses' perceptions of staffing adequacy? J Nurs Adm 2002;32:234-42.

38 Kramer M, Schmalenberg C. Revising the Essentials of Magnetism tool: there is more to adequate staffing than numbers. J Nurs Adm 2005;35:188-98.

39 Aiken LH, Clarke SP, Sloane DM, et al. Effects of hospital care environment on patient mortality and nurse outcomes. J Nurs Adm 2008;39(7-8 Suppl):S45-51.

40 McHugh MD, Ma C. Hospital nursing and 30-day readmissions among medicare patients with heart failure, acute myocardial infarction, and pneumonia. Med Care 2013;51:52-9.

41 Aiken LH, Sermeus W, Van den Heede K, et al. Patient safety, satisfaction, and quality of hospital care: cross sectional surveys of nurses and patients in 12 countries in Europe and the United States. BMJ 2012;344:e1717. 